\title{
Anomalies in the Charge Yields of Fission Fragments from the ${ }^{238} \mathbf{U}(n, f)$ Reaction
}

J. N. Wilson, ${ }^{1}$ M. Lebois, ${ }^{1}$ L. Qi,${ }^{1}$ P. Amador-Celdran, ${ }^{2}$ D. Bleuel, ${ }^{3}$ J. A. Briz, ${ }^{4}$ R. Carroll, ${ }^{5}$ W. Catford,${ }^{5}$ H. De Witte, ${ }^{6}$ D. T. Doherty, ${ }^{7}$ R. Eloirdi, ${ }^{2}$ G. Georgiev, ${ }^{8}$ A. Gottardo, ${ }^{1}$ A. Goasduff, ${ }^{8}$ K. Hadyńska-Klęk, ${ }^{9}$ K. Hauschild, ${ }^{8}$ H. Hess, ${ }^{10}$ V. Ingeberg, ${ }_{11}^{11}$ T. Konstantinopoulos, ${ }^{8}$ J. Ljungvall, ${ }^{8}$ A. Lopez-Martens, ${ }^{8}$ G. Lorusso, ${ }^{12}$ R. Lozeva, ${ }^{8}$ R. Lutter, ${ }^{13}$ P. Marini, ${ }^{14}$ I. Matea, ${ }^{1}$ T. Materna,${ }^{7}$ L. Mathieu, ${ }^{15}$ A. Oberstedt, ${ }^{16}$ S. Oberstedt, ${ }^{17}$ S. Panebianco, ${ }^{7}$ Zs. Podolyák, ${ }^{5}$ A. Porta, ${ }^{4}$ P. H. Regan ${ }^{5,12}$ P. Reiter, ${ }^{10}$ K. Rezynkina, ${ }^{6}$ S. J. Rose, ${ }^{11}$ E. Sahin, ${ }^{11}$ M. Seidlitz, ${ }^{10}$ O. Serot, ${ }^{18}$ R. Shearman, ${ }^{5,12}$ B. Siebeck, ${ }^{10}$ S. Siem, ${ }^{11}$ A. G. Smith, ${ }^{19}$ G. M. Tveten, ${ }^{11}$ D. Verney, ${ }^{1}$ N. Warr, ${ }^{10}$ F. Zeiser,${ }^{11}$ and M. Zielinska ${ }^{7}$

${ }^{1}$ Institut de Physique Nucléaire, CNRS-IN2P3, Université Paris-Sud, Université Paris-Saclay, 91406 Orsay Cedex, France

${ }^{2}$ European Commission, Joint Research Centre, Directorate G for Nuclear Safety and Security, Unit G.I.5, Postfach 2340, 76215 Karlsruhe, Germany

${ }^{3}$ Lawrence Livermore National Laboratory, 7000 East Avenue, Livermore, California 94550, USA

${ }^{4}$ Subatech/Ecole des Mines, 4 rue Alfred Kastler, 44307 Nantes, France

${ }^{5}$ Department of Physics, University of Surrey, Guildford GU2 7XH, United Kingdom

${ }^{6}$ Institute for Nuclear and Radiation Physics, KU Leuven, 3000 Leuven, Belgium

${ }^{7}$ Irfu, CEA, Université Paris Saclay, 91191 Gif-sur-Yvette Cedex, France

${ }^{8}$ CSNSM Orsay, CNRS-IN2P3, Université Paris-Sud, Université Paris-Saclay, 91405 Orsay Cedex, France

${ }^{9}$ INFN Laboratori Nazionali di Legnaro, Viale dell'Universitá, 2, I-35020 Legnaro, Italy

${ }^{10}$ Institut für Kernphysik, Zülpicher Strasse 77, 50937 Köln, Deutschland

${ }^{11}$ University of Oslo, Department of Physics, P.O. Box 1048, Blindern, 0316 Oslo, Norway

${ }^{12}$ National Physical Laboratory, Hampton Road, Teddington, Middlesex TW11 OLW, United Kingdom

${ }^{13}$ LMU München, Schellingstrasse 4, 80799 Munich, Germany

${ }^{14}$ CEA/DAM, DIF, Bruyeres-le-chatel, 91297 Arpajon, France

${ }^{15}$ CENBG Bordeaux, Chemin du Solarium, Le Haut Vigneau, BP 120, 33175 Gradignan, France

${ }^{16}$ Extreme Light Infrastructure-Nuclear Physics (ELI-NP), 077125 Bucharest-Magurele, Romania

${ }^{17}$ European Commission, Joint Research Centre, Directorate G for Nuclear Safety and Security, Unit G.2, 2440 Geel, Belgium

${ }^{18}$ CEA Cadarache, 13108 Saint Paul lez Durance, France

${ }^{19}$ Department of Physics, University of Manchester, Oxford Road, Manchester M13 9PL, United Kingdom

(Received 3 March 2017; published 1 June 2017)

Fast-neutron-induced fission of ${ }^{238} \mathrm{U}$ at an energy just above the fission threshold is studied with a novel technique which involves the coupling of a high-efficiency $\gamma$-ray spectrometer (MINIBALL) to an inversekinematics neutron source (LICORNE) to extract charge yields of fission fragments via $\gamma-\gamma$ coincidence spectroscopy. Experimental data and fission models are compared and found to be in reasonable agreement for many nuclei; however, significant discrepancies of up to $600 \%$ are observed, particularly for isotopes of Sn and Mo. This indicates that these models significantly overestimate the standard 1 fission mode and suggests that spherical shell effects in the nascent fission fragments are less important for low-energy fastneutron-induced fission than for thermal neutron-induced fission. This has consequences for understanding and modeling the fission process, for experimental nuclear structure studies of the most neutron-rich nuclei, for future energy applications (e.g., Generation IV reactors which use fast-neutron spectra), and for the reactor antineutrino anomaly.

DOI: 10.1103/PhysRevLett.118.222501

We report on results which will affect current knowledge at the interface of three separate domains: nuclear fission, nuclear structure, and energy applications. Measurements of fission fragment charge yields have been made using a novel experimental technique of coupling an innovative, inverse-kinematics, fast-neutron source, LICORNE [1,2], to a high-resolution, high-efficiency $\gamma$-ray spectrometer, MINIBALL [3]. This has allowed a detailed spectroscopic study of fission fragments produced via fast-neutroninduced fission of ${ }^{238} \mathrm{U}(n, f)$ for the first time.

Nuclear fission is a complex, dynamical nuclear process and there are still a number of unanswered questions which remain, particularly with regards to the evolution of isotopic fragment yields as a function of excitation energy. Experimental data are crucial to fully understand what drives the fragment split in fission and, in particular, the respective role of neutron and proton shells remains an unresolved issue [4].

Second, neutron-induced fission is obviously important for energy applications since fragment yields influence reactor function through the total energy release, the synthesis of neutron poisons, the decay heat, and the production of long-lived waste. However, existing data on isotopic and charge yields at energies relevant for future 
fast reactors $(\sim 2 \mathrm{MeV})$ are very sparse. Current libraries used in reactor simulations are mainly based on fission modeling and extrapolations from thermal neutron-induced fission [5]. Furthermore, the products of fast fission of ${ }^{238} \mathrm{U}$ in current reactors disproportionately contribute to reactor antineutrino spectra and may affect the measured antineutrino deficit known as the reactor neutrino anomaly $[6,7]$.

Finally, the fission process and its products are also very important for the study of the nuclear structure of neutronrich isotopes. Fission is a reaction mechanism employed at radioactive beam facilities, which use either in-flight or isotope separation on line (ISOL) techniques to study the most neutron-rich nuclei. Both of these techniques have difficulty in gaining spectroscopic information on neutronrich nuclei at moderate or high spin due to either very high fragment recoil velocities and associated Doppler broadening for an in-flight technique or studies that are usually limited to $\beta$ decay for ISOL.

The study of higher spin states of neutron-rich nuclei requires high-resolution spectroscopy of the prompt $\gamma$ decay of slowly moving or stopped fission fragments. One obvious method is to study spontaneous fission. Extensive spectroscopy of the fragments produced with spontaneous fission (SF) sources ${ }^{252} \mathrm{Cf}(\mathrm{SF})$ and ${ }^{248} \mathrm{Cm}(\mathrm{SF})$ were carried out with the Euroball and Gammasphere spectrometers, leading to a wealth of nuclear structure information [8-11]. The $\gamma$-ray spectroscopy technique was also used to measure fragment isotopic distributions from these sources [12,13] and gives information on fragment spin populations, important for understanding the role of angular momentum in fission. However, measuring using a continuous fission source suffers from the difficulty of correcting for isomeric decays [13]. This can lead to yield underestimates for isotopes with isomeric states of lifetimes greater than a few nanoseconds.

More recently, the EXILL experimental campaign using the EXOGAM spectrometer coupled to thermal neutron beams from the ILL reactor has produced prompt spectroscopic information on lighter fissile systems ${ }^{235} \mathrm{U}\left(n_{\text {th }}, f\right)$ and ${ }^{241} \mathrm{Pu}\left(n_{\mathrm{th}}, f\right)$ for nuclear structure studies $[14,15]$. In contrast to fast-neutron-induced fission, the yields of these thermal neutron-induced reactions are well known and have been measured extensively, for example, with the Lohengrin spectrometer [16].

To gain information on the isotopic yields from fission of even-even (fertile) actinides ${ }^{232} \mathrm{Th}(n, f)$ and ${ }^{238} \mathrm{U}(n, f)$ is technically challenging since a collimated, high-flux fastneutron source is required. These two reactions attract additional interest due to the higher average fragment $N / Z$ ratio $(\sim 1.57)$ after neutron emission, assuming low-energy fission, than those of either thermal neutron-induced or spontaneous fission $(\sim 1.54)$. This makes them ideal for studying the nuclear structure of the most neutron-rich nuclei.
Recently, the aforementioned technical problem has been solved with the development of the LICORNE neutron source at the ALTO facility of the IPN Orsay. Intense ${ }^{7} \mathrm{Li}$ beams from the Tandem accelerator are used to initiate the $p\left({ }^{7} \mathrm{Li}, n\right)^{7} \mathrm{Be}$ inverse reaction to produce quasimonoenergetic neutrons. These are produced with a combination of high flux and natural directionality, emitted in narrow cones at energies of around $2 \mathrm{MeV}$ in the laboratory frame. When coupled to a high-efficiency Ge spectrometer, the neutrons can cleanly exit without directly hitting the sensitive detectors, thus allowing precision spectroscopy of fast-neutron-induced reactions on samples placed in the beam.

In this Letter we report on first measurements of the charge yields for the ${ }^{238} \mathrm{U}(n, f)$ reaction at low energy using this innovative technique. Measurements on mass yields only for ${ }^{238} \mathrm{U}(n, f)$ were made in the 1970 s, principally using the techniques of radiochemistry to separate isotopes close to stability in each mass chain after irradiation of a sample with neutrons [17].

However, the only existing data on charge yields come from prompt spectroscopy of two isotope chains [18], and more recently from an approach using x-ray spectroscopy [19].

The LICORNE directional neutron source in this experiment used a primary beam of ${ }^{7} \mathrm{Li}$ of $16.75 \mathrm{MeV}$ bombarding energy with a beam current of $32 \mathrm{nA}$ pulsed with a 400 ns period and $\sim 2$ ns duration.

The beam was incident on the LICORNE hydrogen gas cell $3.5 \mathrm{~cm}$ long and with a $\mathrm{H}_{2}$ pressure of $1.5 \mathrm{~atm}$ separated from the beam line vacuum by a very thin tantalum foil of $2.7 \mu \mathrm{m}$ and surrounded by a thick $\mathrm{Pb} / \mathrm{Cu}$ cylindrical collimator designed to strongly attenuate $478 \mathrm{keV} \gamma$ rays from Coulomb excitation of the ${ }^{7} \mathrm{Li}$ beam in the cell (see Fig. 1). A neutron spectrum, constrained by the kinematics

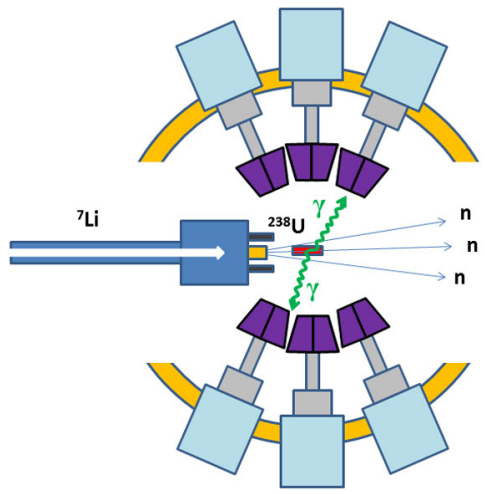

FIG. 1. Schematic diagram of the experimental coupling of the LICORNE directional neutron source with the MINIBALL Ge spectrometer. The main components are the high intensity ${ }^{7} \mathrm{Li}$ beam from the Tandem accelerator; the gas cell with a thin tantalum entrance window surrounded by a $\mathrm{Pb}$ collimator and a $\mathrm{Pb}$ beamstop; the sample, placed directly in the neutron beam; and the Ge detectors of MINIBALL surrounding the sample. 
of the reaction between 0.7 and $3.0 \mathrm{MeV}$, was produced in the gas cell with a mean energy $1.72 \mathrm{MeV}$ and a spread at half maximum of approximately $1 \mathrm{MeV}$. Fluxes of around $2.0 \times 10^{6} \mathrm{n} / \mathrm{s} \mathrm{cm}^{2}$ at the center of the MINIBALL array were estimated from GEANT IV [20] simulations previously validated via ionization chamber measurements at short distances $(8-20 \mathrm{~cm})$ from the source. The maximum neutron cone opening angle was $19.5^{\circ}$ to allow clean escape of the neutrons from the spectrometer. A $31.35 \mathrm{~g}$ depleted uranium sample $\left(99.8 \%{ }^{238} \mathrm{U}\right)$ in the shape of a half cylinder of radius $0.57 \mathrm{~cm}$ and length $2.98 \mathrm{~cm}$ was placed along the beam axis at $1.5 \mathrm{~cm}$ from the end of the gas cell in the center of the MINIBALL spectrometer. This detector is normally based at the Isolde facility at CERN, but on loan to the IPN Orsay from the MINIBALL Collaboration.

MINIBALL consisted of 24 large-volume germanium crystals arranged in eight triple clusters at a distance of $14 \mathrm{~cm}$ from the array center, covering around $33 \%$ of $4 \pi \mathrm{sr}$ in solid angle. The triple clusters were placed in a ringlike configuration as near as possible to $90^{\circ}$ from the beam axis (see Fig. 1). Since the crystals have no anti-Compton suppression, the peak-to-total ratio was low, measured to be 0.22 at $1.33 \mathrm{MeV}$ after using a complex in-cluster and cross-cluster add-back algorithm for neighboring crystals.

The total full energy peak efficiency of MINIBALL was determined to be $5.6 \%$ at $1.33 \mathrm{MeV}$. The sample was irradiated over a period of 11 days of effective beam time, and prompt $\gamma$ rays detected in coincidence were recorded to disk. A coincidence event was defined by applying a trigger condition of $M_{\gamma} \geq 3$ different triple-cluster detectors firing within $1.2 \mu$ s in order to keep the event data acquisition rate to a manageable $\sim 5 \mathrm{kHz}$ and the dead time to less than $10 \%$. Each germanium crystal was typically firing at a rate of $8 \mathrm{kHz}$, close to the maximum rate achievable without significant pileup.

It was estimated that the fission rate in the uranium sample was $\approx 25 \mathrm{kHz}$, based on the available neutron flux, the known fission cross section, and the neutron energy spectrum. The average neutron energy inducing fission events was $1.97 \mathrm{MeV}$. During the experiment, $2.8 \times$ $10^{9} M_{\gamma} \geq 3$ coincidences were collected and time referenced to the beam pulsing, giving a large data set rich in both prompt and delayed $\gamma$-ray correlations.

Independent yields for detectable even-even isotopes were deduced from measuring the intensities of all observable transitions feeding each $2^{+}$state, coincident with the corresponding $2^{+} \rightarrow 0^{+}$transition. Figure 2 shows typical spectra observed when gating on low-lying neutron-rich isotopes in the tellurium chain.

The measurement of yields was restricted to the subset of 40 even-even isotopes for two reasons: First, the odd isotopes often have complicated decay schemes with the decay to the ground state fragmented over many pathways, making it difficult to perform a reliable intensity sum.

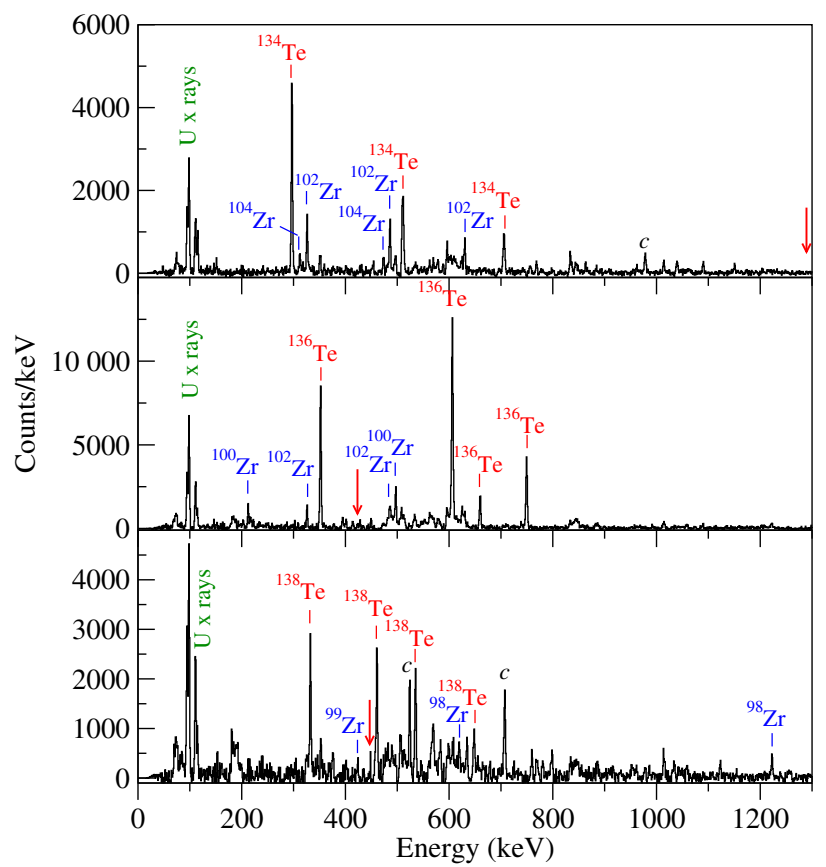

FIG. 2. Typical $\gamma$-ray spectra produced from gating on lowlying transitions, particularly fission products, in this case the neutron-rich even-even nuclei in the Te isotope chain. Coincident $\gamma$ rays from the same nucleus are marked in red, along with $\gamma$ rays from the most prominent binary partners in the $\mathrm{Zr}$ isotope chain. The positions of the gating transitions are marked with red arrows for the 1280,461 , and $443 \mathrm{keV}$ low-lying transitions in ${ }^{134} \mathrm{Te}$, ${ }^{136} \mathrm{Te}$, and ${ }^{138} \mathrm{Te}$, respectively.

Second, these nuclei also often contain many low-energy transitions where measured intensities have large uncertainties in this particular experiment due to the rapid drop in efficiency at low energy caused by the self-shielding of the uranium sample itself coupled to the background of Compton-scattered $\gamma$ rays centered around $250 \mathrm{keV}$ in the unsuppressed MINIBALL spectrometer.

To obtain yields required correction of the measured $\gamma-\gamma$ coincidence intensities for the absolute detection efficiency of MINIBALL, including the self-shielding effects of the ${ }^{238} \mathrm{U}$ sample - a factor of 2.2 at $400 \mathrm{keV}$ and a factor of 5.2 at $200 \mathrm{keV}$. The efficiency as a function of $\gamma$-ray energy was determined from a combination of MCNP [21] simulations of MINIBALL with the uranium sample and direct measurements from cascades below known isomeric decays in particular fission fragments (e.g., ${ }^{132,134,135} \mathrm{Te}$ ) [22].

Since the LICORNE fast-neutron beam was pulsed with a $400 \mathrm{~ns}$ period, corrections to measured yields could be made due to the presence of isomeric states. In fragments with isomers of half-lives greater than a few nanoseconds, the lower-lying transitions are detected promptly only if fed by transitions which bypass the isomer. Without these corrections, measured yields for nuclei with isomers would be significantly underestimated, typically by factors of 2 to 3. Correction required measuring the intensities of delayed 
$\gamma$ rays between successive beam bursts from isotopes such as ${ }^{130,132} \mathrm{Sn},{ }^{132,134,135} \mathrm{Te}$, etc., and deducing the missing yields from the known isomer half-lives.

Statistical errors on the final deduced yields come from the fluctuations of the counts in the observed peaks and the backgrounds on which they sit. In general, the error bars are significantly smaller for spherical nuclei near closed shells due to the high energies of their excited states. For mid shell, deformed nuclei with lower excited states (e.g., Ba and $\mathrm{Ce}$ ), statistical errors are much greater due to the dropping efficiency and increasing background for $\gamma$ rays at lower energies. Potential systematic errors may arise from the MINIBALL efficiency calibration, which included the self-shielding of the sample. These errors are typically around 5\%, but increasing to as much as $20 \%$ for energies below $200 \mathrm{keV}$. Another potential source of systematic error comes from the absolute normalization of the yields. This normalization can be carried out only by assuming that there are no aggregate odd-even effects and that even-even isotopes account for $25 \%$ of the total yield. The spectroscopic technique for measuring yields has two other associated difficulties which may limit its precision: first, the inability to measure the small fraction of direct feeding of the $0^{+}$state, which bypasses the $2^{+}$one and may vary between isotopes; second, a possible incomplete sum of all of the branches which feed the $2^{+}$state, especially via weak, unobservable feeding transitions.

The complete set of yield measurements for the detectable even-even isotopes produced in the experiment can be seen in Fig. 3. The solid lines represent double-Gaussian fits to each fragment pair (e.g., $\mathrm{Zr} / \mathrm{Te}$ ) with five free parameters: widths, heights, and positions could vary, but the areas of the two Gaussians were constrained to be equal, thus assuming charge conservation and no proton emission

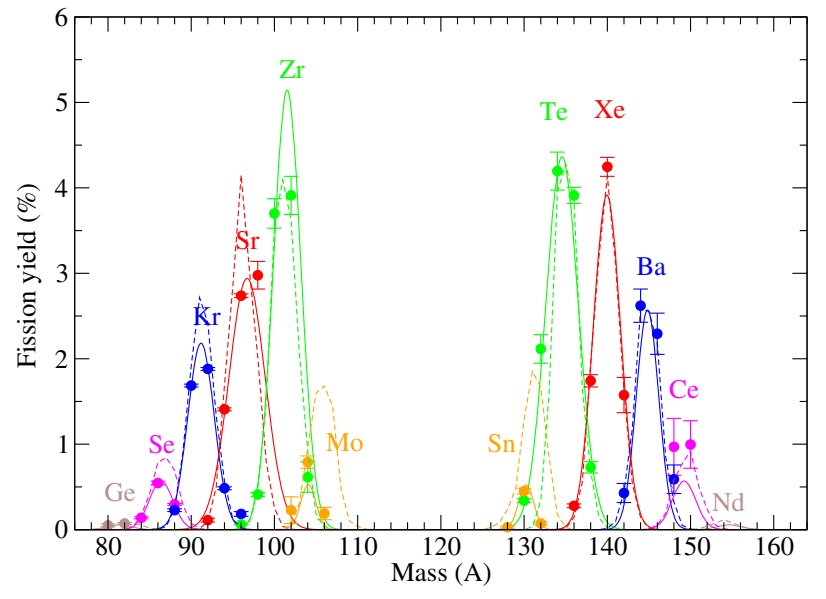

FIG. 3. The complete set of isotopic yield measurements for even-even nuclei observed in the experiment. The solid lines are double-Gaussian fits to the experimental data for each element pair (e.g., $\mathrm{Zr} / \mathrm{Te}$ ). The dashed lines are the predictions of the JEFF3.1.1 evaluated data library [5]. from the deexciting fragments. For the pairs with fewer data points available, the number of free fit parameters was correspondingly reduced (e.g., for the Mo/Sn pair, widths were also constrained to be equal and only four free parameters were used). The dashed lines show the expected yields from the JEFF3.1.1 library [5], which is based on the fission model used by the JEFF evaluators since data at incident neutron energies near $2 \mathrm{MeV}$ are almost nonexistent. From the figure, it can be seen clearly that there are significant discrepancies in both the positions and the sizes of the isotopic yield curves between evaluation (model) and experiment. The discrepancies can be seen more clearly in Fig. 4, where we obtain the charge yield and the average $N$ for a given $Z$ by integrating and analyzing the experimental fitted curves, extracting the appropriate error bars from the residuals of the fit.

The measured charge yields for a given $Z$ are compared to the JEFF3.1.1 [5] and ENDF.B7v1 [23] evaluations or models and the statistical fission model, GEF [24], and can be seen in Fig. 4. The models or evaluations reproduce the measured charge yields with a reasonable accuracy (20\%) except for the Mo/Sn isotope pair, which represents a huge discrepancy of $500 \%-600 \%$.

Figure 5 shows measurements of the position of the average yield for a given charge, $Z$, showing how many fewer neutrons the postemission fragments (on average) possess than the number expected if we use the $N / Z$ ratio of the fissioning compound nucleus, in this case, ${ }^{239} \mathrm{U}$ $(N / Z=1.598)$. Again, comparisons with GEF, JEFF3.1.1, and ENDF.B7v1 are made. The models all reproduce the sawtooth structure, which looks similar to the structure observed for fission neutron multiplicities, in correlation with the fragment mass [25].

The dip at $Z=50$ is clearly caused by the closed spherical shell. The position is reproduced to typically

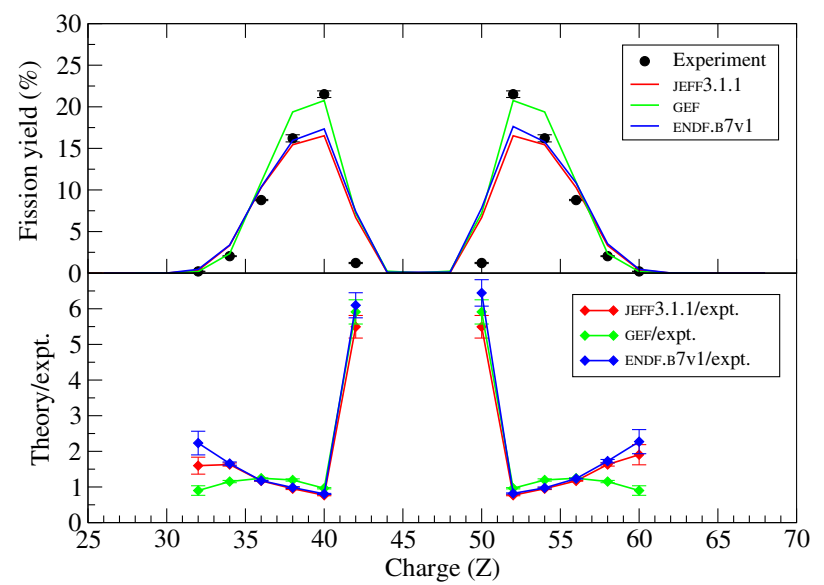

FIG. 4. (Top panel) The measured charge yields for even- $Z$ isotopes produced in the ${ }^{238} \mathrm{U}(n, f)$ reaction compared to the JEFF3.1.1 and ENDF.B7v1 libraries [5,23] and the GEF code [24]. (Bottom panel) The ratio of theory to experiment. 


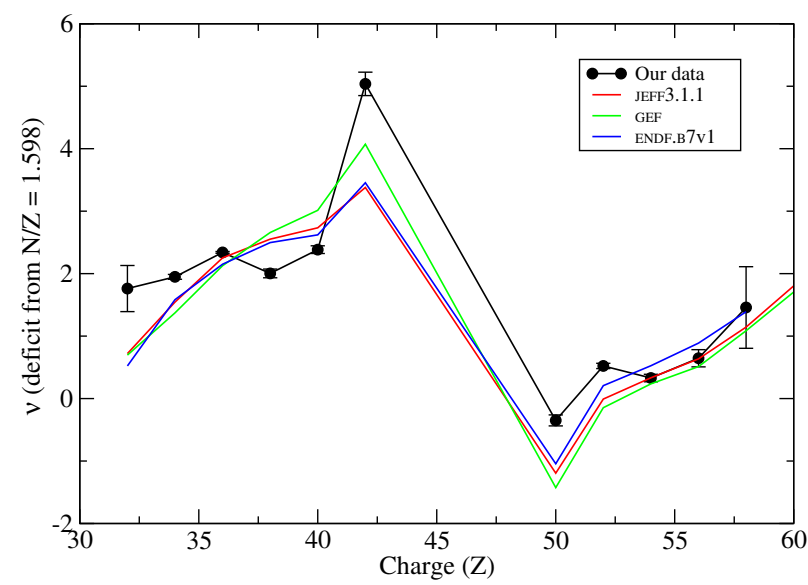

FIG. 5. The deficit in the number of neutrons, $\nu$, of the average fission fragment from the $N / Z$ ratio of the ${ }^{239} \mathrm{U}^{*}$ compound nucleus for the even- $Z$ nuclei studied in the experiment.

0.3 neutrons or less; however, in the more extreme cases (again, Mo and Sn), the models predict fragments that are, on average, $\approx 1$ to 2 neutrons more neutron rich than those measured experimentally. Again, this is an additional piece of evidence suggesting that the models are overestimating the role of spherical shell effects in fission at these excitation energies.

The observed discrepancies between the experiment and the models highlight the difficulties in reproducing experimental data in regions where the data are very sparse. Predictions of isotopic yields from well-measured systems using thermal neutron-induced fission [e.g., ${ }^{235} \mathrm{U}\left(n_{\text {th }}, f\right)$ and ${ }^{239} \mathrm{Pu}\left(n_{\text {th }}, f\right)$ ] are highly accurate since model parameters are tuned to reproduce these data.

The main theoretical understanding of the mass split in the fission process is in terms of different fission modes which describe the path to fission over a complicated potential energy landscape, defined as a function of the deformation parameters of the fissioning system. The troughs and valleys in this landscape are caused by both spherical and deformed shell effects in the nascent fragments, to which the fissioning system is attracted. The modes are typically called standard $1(S 1)$, standard $2(S 2)$, and superlong in the Brosa model [26]. The $S 1$ mode is thought to be associated with one spherical heavy fragment near doubly magic ${ }^{132} \mathrm{Sn}$ and a moderately deformed light fragment. The $S 2$ mode is associated with a heavy deformed fragment of around mass $A \approx 140$ near the deformed $N \approx 88$ shell, and a moderately deformed light fragment. The superlong mode has a low total kinetic energy release and is associated with symmetric fission, and it thus begins to play a significant role only at much higher excitation energies (typically $>10 \mathrm{MeV}$ ).

Our ${ }^{238} \mathrm{U}(n, f)$ charge yield experimental data suggest an overestimation of the $S 1$ mode and thus an overestimation of the importance of spherical shell effects at scission. Since so little information on charge yields in fission is available at these energies, the observed phenomenon (overestimation of the $S 1$ mode and importance of the spherical shell effects) could prove a more general feature of neutron-induced fission at these energies. It would thus have broader consequences for fast-neutron-induced fission at energies relevant for Generation IV reactors and thus have a larger impact on simulations and designs. Alternatively, our reported observations could be an effect confined to the peculiarities of the ${ }^{239} \mathrm{U}^{*}$ fissioning system, even though the microscopic shell effects in the nascent fragments should affect all fissioning systems at such energies. Further investigations are clearly needed.

The results of our experiment also confirm that the experimental technique-and the ${ }^{238} \mathrm{U}(n, f)$ reaction mechanism in particular-is an excellent method to produce or study some of the most exotic neutron-rich nuclei where few or no excited states are known. For example, nuclei such as ${ }^{141} \mathrm{Te}$ and ${ }^{104} \mathrm{Sr}$ appear to be sufficiently well populated as to be accessible to study with a suitable Compton suppressed spectrometer. With this new technique, extra selectivity is gained due to the neutron beam pulsing, which allows gating on delayed isomeric transitions and suppresses the $\beta$-decay background which accounts for approximately half of the $\gamma$ rays emitted in fission. Nuclear structure information on specific isotopes will be the subject of future publications. Furthermore, improvements to the technique are currently under way.

Charge yields of even- $Z$ elements in the ${ }^{238} \mathrm{U}(n, f)$ reaction have been measured using a new technique by coupling a powerful $\gamma$-ray spectrometer to the newly developed LICORNE directional neutron source. The measured yields have been compared with the JEFF3.1.1 and GEF models, which reproduce most yields to within $20 \%$. However, for the Sn/Mo element pair, discrepancies of up to $600 \%$ are observed. One possible explanation is an overestimation of the strength of the $S 1$ mode in the models, and hence an overestimation of the importance of spherical shell effects in fission at these excitation energies. The results have consequences for fission modeling, particularly at energies of around $2 \mathrm{MeV}$ relevant for energy applications. The technique used will also be an important future tool for the study of the nuclear structure of neutron-rich isotopes at moderate spins.

We would like to thank the staff at the ALTO facility for the production of high intensity ${ }^{7} \mathrm{Li}$ beams, the German BMBF under Contract No. 05P15PKCIA (ISOLDE) and Verbundprojekt 05P2015, the Research Council of Norway under Project Grant No. 222287, and the Science and Technology Facilities Council (STFC) funding agency, UK.

[1] M. Lebois, J. N. Wilson, P. Halipré, B. Leniau, I. Matea, A. Oberstedt, S. Oberstedt, and D. Verney, Nucl. Instrum. Methods Phys. Res., Sect. A 735, 145 (2014). 
[2] J. N. Wilson, M. Lebois, P. Halipré, S. Oberstedt, and A. Oberstedt, Phys. Procedia 64, 107 (2015).

[3] N. Warr et al., Eur. Phys. J. A 49, 40 (2013).

[4] C. Böckstiegel, S. Steinhäuser, K.-H. Schmidt, H.-G. Clerc, A. Grewe, A. Heinz, M. de Jong, A. R. Junghans, J. Müller, and B. Voss, Nucl. Phys. A802, 12 (2008).

[5] M. A. Kellett, O. Besillon, and R. W. Mills, OECD Nuclear Energy Agency Report No. 6287, 2009.

[6] A. C. Hayes, J. L. Friar, G. T. Garvey, D. Ibeling, G. Jungman, T. Kawano, and R. W. Mills, Phys. Rev. D 92, 033015 (2015).

[7] P. Huber, Phys. Rev. C 84, 024617 (2011).

[8] J. H. Hamilton, A. V. Ramayya, S. J. Zhu, G. M. TerAkopian, Yu. Ts. Oganessian, J. D. Cole, J. O. Rasmussen, and M. A. Stoyer, Prog. Part. Nucl. Phys. 35, 635 (1995).

[9] J. K. Hwang et al., Phys. Rev. C 57, 2250 (1998).

[10] A. G. Smith et al., Phys. Rev. C 60, 064611 (1999).

[11] T. Rzaca-Urban, W. Urban, J. L. Durell, A. G. Smith, and I. Ahmad, Phys. Rev. C 76, 027302 (2007).

[12] G. M. Ter-Akopian et al., Phys. Rev. C 55, 1146 (1997).

[13] A. Bogachev et al., Eur. Phys. J. A 34, 23 (2007).

[14] G. de France et al., EPJ Web Conf. 66, 02010 (2014).

[15] P. Baczyk et al., Phys. Rev. C 91, 047302 (2015).
[16] H. G. Börner and F. Gönnenwein, The Neutron: A Tool and an Object in Nuclear and Particle Physics (World Scientific, Singapore, 2012).

[17] S. Nagy, K. F. Flynn, J. E. Gindler, J. W. Meadows, and L. E. Glendenin, Phys. Rev. C 17, 163 (1978).

[18] W. R. Phillips, A. P. Byrne, G. D. Dracoulis, G. J. Lane, T. R. McGoram, and R. Newman, Eur. Phys. J. A 3, 205 (1998).

[19] T. Granier, R. O. Nelson, T. Ethvignot, M. Devlin, N. Fotiades, P. E. Garrett, and W. Younes, Eur. Phys. J. A 49, 114 (2013).

[20] S. Agostinelli et al., Nucl. Instrum. Methods Phys. Res., Sect. A 506, 250 (2003).

[21] See https://mcnp.lanl.gov/.

[22] R. E. Sund, H. Weber, and V. V. Verbinski, Phys. Rev. C 10, 853 (1974).

[23] M. B. Chadwick et al., Nucl. Data Sheets 112, 2887 (2011).

[24] K.-H. Schmidt, B. Jurado, C. Amouroux, and C. Schmitt, Nucl. Data Sheets 131, 107 (2016).

[25] A. Göök, F.-J. Hambsch, and M. Vidali, Phys. Rev. C 90, 064611 (2014).

[26] U. Brosa, S. Grossman, and A. Müller, Phys. Rep. 197, 167 (1990). 